\title{
La violencia hacia el adulto mayor y sus consecuencias
}

Violence towards the elderly and its consequences

Edmundo N. Chóez Chiliquinga ${ }^{1}$, María F. Zambrano Pérez ${ }^{2}$, Mayra V. Barros Carvajal $^{3} \&$ Martha M. Parra Parra ${ }^{4}$

Recibido: 10-02-2019 / Revisado: 15-02-209 /Aceptado: 04-03-2019/ Publicado: 14-06-2019

\begin{abstract}
.
DOI: https://doi.org/10.33262/cienciadigital.v3i1.1.362

One of the preventive variants of violence that has greater feasibility of application, lies in the location and protection of elderly. Objective: To characterize the phenomenon of elder abuse reported flagrantly in the period January 2016 to December 2017 in the city of Quito. Methodology: A descriptive epidemiological study was carried out. Results: 219 cases were studied in which male sex prevailed (55.7\%), married marital status $(57.1 \%)$ and primary education $(45.7 \%)$. The aggressions occurred in the afternoon (12:00 to 18:00) (36\%), and in the fourth quarter of the year. The lesions were mild $(85.4 \%)$, produced by the extremities of the aggressor $(53 \%)$ and located in the head $(61.2 \%)$. Conclusions: it was observed that the most vulnerable elderly has low levels of schooling, the aggressors use their extremities to attack them especially in easily accessible places such as the victims' heads.
\end{abstract}

Keywords: Adult, elder, violence, victim, aggressor.

\section{Resumen.}

Una de las variantes preventivas de la violencia que posee mayor factibilidad de aplicación, radica en la localización y protección de los adultos mayores. Objetivo: Caracterizar al fenómeno del maltrato al adulto mayor denunciado en flagrancia en el período enero 2016 a diciembre del 2017 de la ciudad de Quito. Metodología: Se realizó un estudio epidemiológico descriptivo. Resultados: se estudiaron 219 casos en los cuales prevaleció el sexo masculino $(55,7 \%)$, el estado civil casado $(57,1 \%)$ e instrucción primaria $(45,7 \%)$. Las agresiones se presentaron en la tarde (12:00 a 18:00) (36 \%). Las lesiones fueron de carácter leve $(85,4 \%)$, producidas por las

\footnotetext{
${ }^{1}$ Universidad Central del Ecuador. Correo-e: edmundo.choez@gmail.com

${ }^{2}$ Universidad Central del Ecuador. Correo-e: a15 fernanda@hotmail.com

${ }^{3}$ Universidad Central del Ecuador. Correo-e: mvbcmedi7@hotmail.com

${ }^{4}$ Universidad Católica de Cuenca. Correo-e: marthaparra20@hotmail.com
} 
extremidades del agresor (53\%) y localizados en la cabeza $(61,2 \%)$. Conclusiones: se observó que el adulto mayor más vulnerable presenta bajos niveles de escolaridad, los agresores usan sus extremidades para agredirlos sobre todo en lugares de fácil acceso como la cabeza de las víctimas.

Palabras claves: Adulto, mayor, violencia, víctima, agresor.

\section{Introducción.}

La población adulta mayor está en aumento rápido y progresivo a nivel mundial, lo cual asocia del mismo modo un incremento progresivo de violencia hacia este grupo poblacional, incluso en formas silentes, con fuertes implicaciones en la calidad de vida de esta población. Actualmente, la violencia hacia los adultos mayores se ha revelado como un serio problema de salud pública, captando cada vez en mayor medida el interés de nuestra sociedad, particularmente de la comunidad científica. Una de las variantes preventivas y reductoras de la violencia que posee mayor factibilidad de aplicación, radica en la localización y especial protección de los adultos mayores.

Los primeros estudios acerca de la violencia hacia el adulto mayor se remontan a finales de los años sesenta con la publicación de algunos artículos británicos. En las décadas de 1980 y 1990 hubo un desarrollo en la investigación y en la atención del problema del maltrato de personas mayores, principalmente en Estados Unidos y Canadá. Es probable que el incremento en las estadísticas haya incidido también para que más profesionales de diversas disciplinas se interesen en la investigación del tema.

La población mundial está envejeciendo rápidamente. Entre 2015 y 2050 la proporción de la población mundial mayor de 60 años se multiplicará casi por dos, pasando del 12\% al 22\%, la inmensa mayoría de los cuales vivirán en países de ingresos medianos o bajos. En el Ecuador existen: 1.049 .824 personas mayores de 65 años que representa el 6,5\% de la población total. En el año 2020 será del 7,4\%. Para el año 2054 se prevé que representen el 18\% de la población. Para las mujeres la esperanza de vida será mayor con 83,5 años comparado con los 77,6 años de los hombres.

Se define adulto mayor al grupo etario que comprende personas que tienen más de 65 años de edad. Por lo general, se considera que los adultos mayores, sólo por haber alcanzado este rango de edad, se los reconoce como pertenecientes a la tercera edad o ancianos. La Organización Mundial de la Salud define al maltrato hacia el adulto mayor como "Cualquier acto aislado o repetitivo o la falta de acción apropiada, ocurrida en cualquier relación de la que se espera confianza, que causa daño o malestar a la persona mayor" (Montero et al, 2017, p. 3). La Organización Mundial de la Salud en el informe mundial sobre la violencia y la salud 2002 define a la violencia como: "El uso deliberado de la fuerza física o el poder, ya sea en grado de amenaza o efectivo, contra uno mismo, otra persona o un grupo o comunidad, que cause o tenga muchas probabilidades de causar lesiones, muerte, daños psicológicos, trastornos del desarrollo o privaciones" (OMS, 2002, p.15). Según su naturaleza la violencia se determina como: Violencia física. Todo acto de fuerza que cause daño, dolor o sufrimiento físico en las personas agredidas, cualquiera que sea el medio empleado y sus consecuencias, sin considerar el tiempo que 
se requiere para su recuperación. Violencia psicológica. Constituye toda acción u omisión que cause daño, dolor, perturbación emocional, alteración sicológica o disminución de la autoestima de la persona agredida. Es también la intimidación o amenaza mediante la utilización de apremio moral sobre otro miembro de familia infundiendo miedo o temor a sufrir un mal grave e inminente en su persona o en la de sus ascendientes, descendientes o afines hasta el segundo grado. Violencia Sexual. Se considera violencia sexual todo maltrato que constituya imposición en el ejercicio de la sexualidad de una persona y que la obligue a tener relaciones u otras prácticas sexuales con el agresor o con terceros, mediante el uso de fuerza física, intimidación amenazas o cualquier otro medio coercitivo, la violencia sexual incluye al acoso sexual, la violación, al ultraje y los delitos contra la libertad sexual. Violencia Patrimonial o económica. Constituye una forma más de violencia psicológica que, en ocasiones, coarta la libertad física porque impide a la persona el ejercicio de su libertad de movimiento y acción; consiste en privar a la persona de los medios económicos de subsistencia para ella y sus hijos o de afectarla patrimonialmente. Se enfatiza que la violencia hacia el adulto mayor no puede limitarse solo a los aspectos físico, emocional, sexual o patrimonial también existe la violencia financiera, por negligencia, negación a brindarle ayuda y otras formas más, lo cual sería materia de análisis.

Se reporta que el perfil de la víctima es: femenina entre 65-75 años, jubilada, residente en áreas urbanas, aislada socialmente. Los adultos mayores con discapacidad física o mental, tiene aún más riesgo en la medida que tengan limitaciones inherentes en las actividades básicas de vida diaria que los hagan dependientes parciales o totales. Se describe que el perfil del agresor corresponde al esposo o hijo hombre entre 35-45 años, que vive en condiciones de estrés. El agresor más prevalente es la pareja, seguido de hijos, desconocidos, hermanos y vecinos; difícil de identificar pues las víctimas temen reportarlo, por ellos mismos y por proteger a su familia y amigos, por temor de que la situación se torne aún menos favorable y es más prevalente en mujeres casadas.

\section{Metodologia.}

Por las características de los objetivos el presente estudio tiene un diseño de carácter epidemiológico descriptivo. Epidemiológico porque se describe la frecuencia y las características más importantes de un problema de salud. Descriptivo debido a que se recolecta datos que describan la situación tal y como es. El estudio se efectuó en la unidad de flagrancia y las unidades de atención en peritaje integral de la ciudad de Quito (Quitumbe), el universo corresponde a los adultos mayores víctimas de violencia en el período enero 2016 a diciembre del 2017, tomando como fuente de datos los registrados en los informes forenses de lesiones, violencia intrafamiliar y delitos sexuales. Durante el período de dos años existe una población de sujetos de estudio de 219 adultos mayores víctimas de violencia. Por lo cual se decidió tomar para el estudio toda la población, por lo indicado no se realizó ninguna muestra. Se procedió a tomar todos los datos necesarios para la presente investigación, los cuales fueron recolectados en un formulario de datos basado en el formato de informes Médico Legales de la Fiscalía General del Estado. 
Las técnicas utilizadas para la recolección de datos fueron, la revisión y posterior recolección de información necesaria de los informes forenses de lesiones, violencia intrafamiliar y delitos sexuales de los adultos mayores víctimas de violencia que acudieron a Flagrancia y Unidad de Atención en Peritaje Integral de la ciudad de Quito en el período enero 2016 a diciembre 2017. Tras la recolección de la información, se organizó una tabla por categorías en el Programa Microsoft Excel 2010, posterior a su revisión se creó una base de datos que fue codificada para luego ser exportada y analizada en el paquete estadístico SPSS versión 24 de la Facultad de Ciencias Médicas, obteniéndose estadística descriptiva para análisis uni-variado y bi-variado, para esta última se aplicó la prueba de Chi cuadrado, debido a que las variables a asociar son cualitativas.

\section{Resultados.}

Del total de víctimas (N: 219), se observó que el 55,7\% eran hombres y el 44,3\% eran mujeres. El rango de edad estuvo comprendido entre 65 a 92 años y la media de 70,8 años. Con respecto al estado civil el $57,1 \%$ se encontraban casados. En cuanto a la instrucción 45,7\% tenían una instrucción básica. (Ver la tabla 1).

\begin{tabular}{ll}
\hline Grupo (n) 219 & Porcentaje \\
\hline Sexo & \\
Mujeres & $44,3 \%$ \\
Hombres & $55,7 \%$ \\
\hline Edad, media (rango) & $70,8(65-92)$ \\
\hline Estado civil & \\
Casado & $57,1 \%$ \\
Soltero & $9,1 \%$ \\
Viudo & $14,6 \%$ \\
Divorciado & $13,2 \%$ \\
Unión libre & $5,9 \%$ \\
\hline Instrucción & \\
Ninguno & $9,1 \%$ \\
Inicial & $8,2 \%$ \\
Básica & $45,7 \%$ \\
Bachillerato & $16,4 \%$ \\
Superior & $18,3 \%$ \\
\hline
\end{tabular}


Tabla 1. Características sociodemográficas de las víctimas que recibieron atención en Flagrancia y UAPI* de la ciudad de Quito, enero 2016 a diciembre 2017

*UAPI: Unidad de Atención en Peritaje Integral (Quitumbe)

Fuente: Departamento de Estadística, Informes Forenses de Flagrancia y UAPI

Elaboración: Md. Edmundo Chóez

Respecto al parentesco del agresor con la víctima se observó que el 37\% de las víctimas no tenían ningún tipo de parentesco; $15 \%$ eran sus familiares; $15 \%$ eran sus vecinos; $12 \%$ eran sus hijos; y el $12 \%$ eran conocidos. Del total de casos (N: 219), se observó que $96,3 \%$ fueron víctimas de violencia física y 3,7\% fueron víctimas de violencia sexual. Con respecto al lugar de la agresión el lugar de mayor frecuencia de agresión fue en el hogar de la víctima con 49,3\%. Respeto al objeto empleado en la agresión se observó que el objeto empleado en la agresión con mayor frecuencia fueron las extremidades con $53,4 \%$. Respecto a la hora del hecho se observó que la hora de mayor frecuencia fue de 12:00 a 18:00 en el 36\%; en segundo lugar, de 06:00 a 12:00 con el 27\%; en tercer lugar de 18:00 a 00:00 con el 26\% y en cuarto lugar de 00:00 a 06:00 con el 10\%. En relación a los tipos de lesiones presentes posterior a la agresión se observó que el $71 \%$ presentaron lesiones contusas; el $12 \%$ presentaron heridas contusas; el $10 \%$ presentaron heridas cortantes y el 7\% presentaron fracturas. Respecto a los casos que se pudo establecer incapacidad médico legal se observó que el tiempo de incapacidad de mayor frecuencia fue el de menor a 3 días con el 74,5\%. Respecto a la localización anatómica de las lesiones se observó que el $61,2 \%$ presentaron lesiones en la cabeza; el 34,2\% presentaron lesiones en los miembros superiores; el $15,1 \%$ presentaron lesiones en los miembros inferiores; el 10,5\% presentaron lesiones en el tórax; el 2,3\% presentaron lesiones en cuello, región lumbar y glútea respectivamente y el 1,4\% presentaron lesiones en abdomen y genitales respectivamente.

\section{Conclusiones}

- En la presente investigación se describe las características socio demográficas de los adultos mayores víctimas de violencia y de sus agresores, así como el lugar de los hechos y el resultado de la violencia en las víctimas de los casos atendidos en el período enero 2016 a diciembre del 2017 en flagrancia y las Unidades de Atención en Peritaje Integral de la ciudad de Quito.

- Respecto a las características socio demográficas de los adultos mayores víctimas de violencia, observamos que de los 219 casos estudiados, el 55,7\% pertenecen al sexo masculino y el $44,3 \%$ al sexo femenino, se observa un ligero predominio por el sexo masculino. En otros estudios como el de (Maíz F. et al, 2009-2011) se encontró que el $65,71 \%$ corresponden al sexo femenino y el 34,29\% corresponden al sexo masculino. También en otra investigación realizada por (Enamorado J. et al, 2010-2011) determinaron que el 64\% corresponden al sexo femenino y el $36 \%$ al sexo masculino. Evidenciando en los mencionados estudios un predominio por 
el sexo femenino lo cual difiere de nuestro estudio en el que hubo predominio del sexo masculino, debido que la mayor parte de informes forenses fueron de lesiones, por tanto, en este sentido los hombres acudieron sin dudar a recibir atención Médico Legal.

- En la presente investigación se evidencia que el estado civil con mayor frecuencia es el de adultos mayores casados con el $57,1 \%$ estos datos se correlacionan con los estudios mencionados a continuación. En el estudio realizado por (Maíz F. et al, 2009-2011) encontraron que el $80 \%$ de adultos mayores víctimas de violencia estaban casados. Mientras que (Martina M. et al, 2009) en su estudio evidenció que el $50.85 \%$ de adultos mayores estaban casados.

- En cuanto al nivel instrucción con mayor frecuencia es el de adultos mayores con instrucción básica en el 45,7\%. En otros estudios como el de (Maíz F. et al, 20092011) encontraron que el $85 \%$ de las víctimas tenían estudios primarios. También en otra investigación realizada por (Martina M. et al, 2009) se evidenció que el $47.46 \%$ tenían instrucción primaria.

- Respecto a las características de los agresores en relación al parentesco del agresor con la víctima se observó que el 37\% de las víctimas no tenían ningún tipo de parentesco; $15 \%$ eran sus familiares; $15 \%$ eran sus vecinos; $12 \%$ eran sus hijos; y el $12 \%$ eran conocidos. En otros estudios como el de (Maíz F. et al, 2009-2011) encontraron que en más de la mitad de los casos 55,7\% el agresor era hijo de la víctima. En otros estudios como el de (Martina M. et al, 2009) se evidenció que el hijo(a) fue principal agresor con el 52.54\%. Evidenciando en los mencionados estudios un predominio de que el agresor fue el hijo(a) lo cual difiere de nuestro estudio en el que el parentesco de mayor frecuencia fue de las víctimas que no tenían ningún tipo de parentesco, esto es debido a que en el presente estudio se incluyeron casos de violencia intrafamiliar, lesiones y delitos sexuales denunciados por adultos mayores víctimas de violencia.

- Respecto a las características generales de la agresión se observó que 96,3\% fueron víctimas de violencia física y 3,7\% fueron víctimas de violencia sexual. En otros estudios como el de (Maíz F. et al, 2009-2011) encontraron que el $90 \%$ la violencia es de tipo físico. Mientras que (Martina M. et al, 2009) se evidenció que la violencia psicológica con $77.12 \%$ es la que más reportan los adultos mayores, seguido de la violencia física y psicológica con el $16.1 \%$. Se evidencia que en el presente estudio el tipo de violencia con mayor frecuencia es la agresión física y se relaciona con los estudios mencionados, y difiere con otros. En nuestro estudio se toma en cuenta la violencia de tipo físico y sexual, puesto que la violencia psicológica es subjetiva y se encontraba subregistrada, por tal motivo no se la incluyó.

- En la presente investigación se evidencia que objeto empleado en la agresión con mayor frecuencia fueron las extremidades con el $53,4 \%$ y se relaciona con los estudios mencionados a continuación. En el estudio realizado por (Maíz F. et al, 2009-2011) encontraron que en el 83,8\% las agresiones fueron mediante manos y pies. Así como también (Lalanda S. et al, 2005-2013) determinó que en el 72,9\% las agresiones fueron mediante las extremidades. 
- Respecto a la hora del hecho se observó que la hora del hecho de mayor frecuencia fue de 12:00 a 18:00 en el 36\%; en segundo lugar, de 06:00 a 12:00 con el 27\%; en tercer lugar de 18:00 a 00:00 con el 26\% y en cuarto lugar de 00:00 a 06:00 con el 10\%. En otros estudios como el de (Cortés C. et al, 2006) se determinó que la hora de mayor ocurrencia del hecho se presenta particularmente entre las 18:00 y las 23:59 horas. Evidenciando en el mencionado estudio un predominio entre las horas de tarde y noche lo cual difiere de nuestro estudio en el que hubo predominio de las horas de la tarde, puesto que en nuestro medio en las horas de la tarde la mayor parte de las personas retornan de instituciones educativas, trabajos, entre otros a su hogar.

- Respecto a las características generales de las lesiones su mecanismo y severidad. En la presente investigación se observó que el tipo de lesión con mayor frecuencia fueron las lesiones contusas con el $71 \%$. Se evidencia que en el presente estudio la severidad de lesión con mayor frecuencia fue de carácter leve es decir con una incapacidad médico legal menor a 3 días con el 74,5\% y se relaciona con el estudio mencionado. En el estudio de (Maíz F. et al, 2009-2011) encontraron que el 84\% de los casos las lesiones fueron de carácter leve.

- En la presente investigación se evidencia que la localización anatómica de las lesiones con mayor frecuencia fue en la cabeza con el $61,2 \%$ y se relaciona con el estudio mencionado. En el estudio de (Lalanda S. et al, 2005-2013) se determinó que las ubicaciones preferidas fueron la cabeza y cuello en el $75 \%$.

- Se puede concluir que el fenómeno del maltrato al adulto mayor está descrito en la literatura, pero en la ciudad de Quito no existe un estudio que lo demuestre, en este sentido, en la presente investigación se estudiaron 219 casos de adultos mayores víctimas de violencia tanto física, intrafamiliar y sexual, que acudieron a Flagrancia y las Unidades de Atención en Peritaje Integral de la ciudad de Quito en el período enero 2016 a diciembre del 2017.

- Los adultos mayores que son víctimas de violencia y que acudieron a flagrancia y a las unidades de atención en peritaje integral de la ciudad de Quito en el período enero 2016 a diciembre del 2017, en su mayoría fueron hombres (55,7\%), casados, con bajo nivel de escolaridad. Los agresores de los adultos mayores en su mayoría no tuvieron ningún parentesco con la víctima (vecinos, conocidos). Las agresiones se llevaron a cabo con más frecuencia en el hogar de la víctima, en las horas de la tarde (12:00 a 18:00) y sobre todo en el cuarto trimestre del año. La violencia física fue la más frecuente $(96,3 \%)$ vs. Violencia sexual $(3,7 \%)$. La mayor parte las lesiones físicas fueron contusas de carácter leve (93\%), debido a un mecanismo traumático producto del uso de las extremidades, las cuales provocaron un daño predominante en la cabeza y extremidades del adulto mayor.

- Se recomienda instaurar información respecto al presunto agresor como sexo, edad, estado civil, consumo de drogas y alcohol previo a la agresión en el informe forense de lesiones. Instaurar información respecto a la víctima como ocupación, consumo de drogas y alcohol previo a la agresión en el informe forense de lesiones. Dar seguimiento a los adultos mayores víctimas de violencia para un 
manejo integral y oportuno. Confirmar que todos los datos del informe forense se encuentren llenos.

\section{Referencias Bibliográficas}

Adams J. Maltrato en el adulto mayor institucionalizado. Revista Médica Clínica Las Condes. 2012 enero; 23(1).

Aguilar V, Fuentes Y, Medel V. Prevalencia de sospecha de maltrato en el adulto mayor y factores vinculados. 2014. Tesis de especialidad, Universidad Veracruzana. Available from: https://www.uv.mx/blogs/favem2014/files/2014/06/Tesis1.pdf

Camacho G. La Violencia de género contra las mujeres en el Ecuador. Quito: Consejo Nacional para la Igualdad de Genero; 2014. Report No.: 978-9942-07-761-5. Available from: https://www.unicef.org/ecuador/Violencia_de_Gnero.pdf

COIP. Código Orgánico Integral Penal. 2014. Asamblea Nacional de la República del Ecuaro, Registro Oficial Suplemento 180.

Cortés C, Soriano M. Violencia Intrafamiliar Colombia. 2006. Instituto Nacional de Medicinal Legal y Ciencias Forenses. Available from: http://www.medicinalegal.gov.co/documents/20143/49496/Violencia+Intrafa miliar.pdf

Enamorado G, Pérez J, Domínguez A. Violencia intrafamiliar contra el adulto mayor en una comunidad de Guinea Bissau. Scielo. 2013 julio; 7(7). Available from: http://scielo.sld.cu/scielo.php?script=sci_arttext\&pid=S102930192013000700004

Giraldo Rodríguez L. Maltrato de personas mayores. Medigraphic. 2010 mayo; 5(2).

Krug E, Dahlberg L. Informe mundial sobre la violencia y salud. Informe mundial. Washington, D.C.: OPS, OMS, Oficina Sanitaria Panamericana, Oficina Regional de la OMS; 2003. Report No.: 927531588 4. Available from: https://fiapam.org/wp-content/uploads/2014/03/El-maltrato-a-las-personasmayores.pdf

Lalanda S, Santos M, Norton P. Domestic violence against elderly with disability. Journal of Forensic and Legal Medicine. 2014 septiembre; 28.

Maíz F, Jaurrieta S. Valoración médico forense del maltrato intrafamiliar a ancianos en la subdirección de Bizkaia del Instituto Vasco de Medicina Legal durante el trienio 2009-2011. Scielo. 2012 diciembre; 18(3-4).

Martina M, Nolberto V, Miljanovich M, Bardales O, Gálvez D. Violencia hacia el adulto mayor: Centros Emergencia Mujer. Revista Peruana de Epidemiología. 2010 diciembre; 14(3).

MIES. Agenda de igualdad para Adultos Mayores. [Online].; 2012 [cited 2017 septiembre 28. Available from: https://fiapam.org/wpcontent/uploads/2013/06/Agendas_ADULTOS.pdf. 


\section{Para citar el artículo indexado.}

Chóez E., Zambrano M., Barros M., \& Parra M. (2019) La violencia hacia el adulto mayor y sus consecuencias. Revista electrónica Ciencia Digital 3(1.1), 99-107. Recuperado desde: http://cienciadigital.org/revistacienciadigital2/index.php/CienciaDigital/article/view/362/780

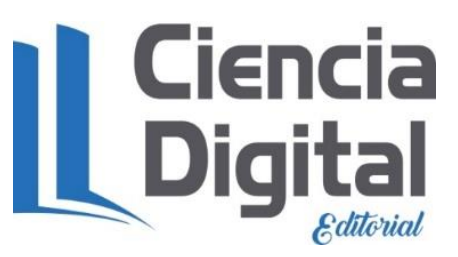

El artículo que se publica es de exclusiva responsabilidad de los autores y no necesariamente reflejan el pensamiento de la Revista Ciencia Digital.

El articulo queda en propiedad de la revista y, por tanto, su publicación parcial y/o total en otro medio tiene que ser autorizado por el director de la Revista Ciencia Digital.
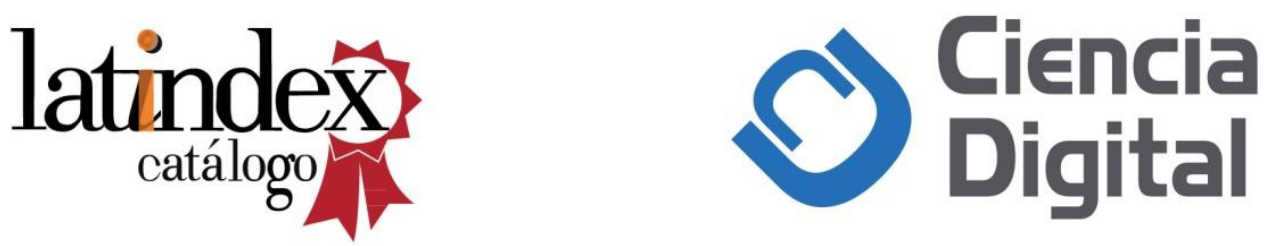